\title{
Impact of Satellite on TWSTFT Accuracy
}

\author{
Hongwei Sun and Yuli Li \\ Xi'an University, Kejiliulu 1, Xi’an, 710065, Shaanxi, China
}

\begin{abstract}
In this paper, the impact of the satellite on two-way satellite time and frequency transfer (TWSTFT) in Asia links is analyzed. If two distances from two stations to the satellite are different, the non-reciprocal paths are produced, which impact the accuracy of TWSTFT. In addition, due to the Earth's rotation and the finite signal velocity, the non-reciprocal paths are also produced, and they mainly result from the aviation of the satellite longitude and altitude.
\end{abstract}

\section{Keywords-satellite motion; TWSTFT; non-reciprocal path}

\section{INTRODUCTION}

Satellite is indispensable for time and frequency transfer of the highest precision and accuracy over large distances, because this cannot be achieved by terrestrial communication techniques. TWSTFT technique via the geo-stationary communication satellite is the greatest prospect in the remote and precise time and frequency transfer. In addition to some TWSTFT links established in Europe and America, several links in Asia region have also been constructed using the communication satellite located $150^{\circ} \mathrm{E}$.

Nowadays, the accuracy of the technique is towards as high as $1 \mathrm{~ns}$, therefore the impact of the systematic errors on accuracy must be studied in detail. A main factor affecting accuracy is satellite motion.

\section{IMPACT OF SATELLITE Motion ON ACCURACY}

TWSTFT is used for point-to-point time transfer. Laboratories do this in pairs, and each laboratory in a pair accesses a transmitting/receiving earth station in order to send its timing information to the other laboratory in the pair via a geo-stationary communication satellite using pseudo-noise-coded signals. The measurement consists of simultaneous time-interval measurements at both sites in which the pulse-per-second (1PPS) signal generated by the local clock starts a local time-interval counter. The signal is also transmitted via the satellite to the other laboratory where it stops the time-interval counter at that site. The principle of TWSTFT is set out in $[1,2]$.

The time difference between the clocks of stations 1 and 2 is given by:

$$
\mathrm{T} 1-\mathrm{T} 2=(\Delta \mathrm{T} 1-\Delta \mathrm{T} 2) / 2+[(\mathrm{TR} 1+\mathrm{RE} 2)]-(\mathrm{TR} 2
$$
+ RE1) $] / 2+($ STR1 - STR2) / 2+ Sagnac effect+ [(DT1 DR1) - (DT2 - DR2)] / 2.

The terms on the right in (1) are grouped according to their nature and are explained as follows.

The first term, ( $\Delta \mathrm{T} 1-\Delta \mathrm{T} 2) / 2$, is the difference in the counter readings of stations 1 and 2 . This difference is exchanged to enable the difference in clocks to be computed.
The second term, [(TR1 + RE2)] - (TR2 + RE1) / 2, is the difference in the sum of the signal delays in the uplinks and downlinks in both signal directions. Under the assumption of path reciprocity, this term would be zero. However, the signals penetrate the Earth's atmosphere, so a non-reciprocal path is taken due to the different uplink and downlink frequencies used.

The third term, (STR1 - STR2) / 2, is the difference of the satellite transponder delays for the signal from station 1 to station 2 and vice versa. When the same frequency band of one transponder is used for both signal directions, the delays are equal and the difference is zero.

The fourth term is a correction for the path non-reciprocity caused by the Earth's rotation (the Sagnac effect). It can be computed from the positions of the Earth stations and the satellite with general knowledge of their positions.

The last term, [(DT1 - DR1) - (DT2 - DR2)] / 2, is the difference of the delay differences between the transmitting and receiving parts of stations 1 and 2 .

As mentioned above, the contribution from the satellite transponder is zero because the same frequency band of one transponder is used for both signal directions in the Asia-Pacific region, so the factors affecting the accuracy are satellite motion, the Earth's atmosphere and the ground station equipment.

In addition to the TWSTFT links among the major time-keeping laboratories in North America and Europe, TWSTFT links have being constructed in the Asia-Pacific region under the leadership of NICT (National Institute of Information and Communications Technology, Japan). They link several important time laboratories: NICT, NMIJ (National Metrology Institute of Japan), TL (Telecommunication Laboratories, Chinese Taipei), NTSC (National Time Service Center, China), NML (National Measurement Laboratory, Australia), SPRING (Productivity and Standards Board, Singapore), and KRISS (Korea Research Institute of Standards and Science, Korea). The NICT-NMIJ, NICT-TL, NICT-NTSC, NICT-SPRING, and NICT-KRISS links use a common geo-stationary satellite located at $150^{\circ} \mathrm{E}$. The NICT-NML link uses a geo-stationary satellite located at $177^{\circ} \mathrm{E}$.

The satellite used in TWSTFT is a geo-stationary satellite. Generally speaking, its motion mainly includes daily periodical motion and drift motion. To some extend, the distance from the station to the satellite can be considered as a sinusoid with amplitude and period of $24 \mathrm{~h}$ : 


$$
R=R_{0}+R_{m} \cdot \sin \left(\frac{2 \pi t}{24}+\phi\right)
$$

where $R O$ is the normal distance from the station to the satellite, $R m$ is the amplitude of the daily change, and $\phi$ is the initial phase. The variation of the distance is:

$$
\frac{d R}{d t}=R_{m} \cdot \cos \left(\frac{2 \pi t}{24}+\phi\right) \cdot \frac{\pi}{12}
$$

Then the greatest rate of variation should be:

$$
\frac{d R}{d t} \leq R_{m} \cdot \frac{\pi}{12}
$$

According to the various greatest amplitudes, we can get the greatest velocities of satellite motion relative the ground. Table I gives some results.

TABLE I. VELOCITY OF SATELLITE RELATIVE GROUND

\begin{tabular}{|c|c|}
\hline$R m \quad(\mathrm{~km})$ & $d R / d t \quad(\mathrm{~m} / \mathrm{s})$ \\
\hline 30 & $<2.18$ \\
\hline 60 & $<4.36$ \\
\hline 90 & $<6.54$ \\
\hline
\end{tabular}

Errors, relating to two aspects, result from the satellite motion. Since the two distances from two stations to the satellite are different, the time taken by the two signals from two stations to reach the satellite is different. Thus the satellite receives the two signals at different positions, which is shown in Fig. 1 (where G1 and G2 denote the two stations, and S1 and S2 denote the positions of the satellite).

An extreme case is with one station having an elevation of $6^{\circ}$ and the other having an elevation of $90^{\circ}$. Thus the greatest difference from the two stations to the satellite is $5893 \mathrm{~km}$ (see Fig. 2). The corresponding greatest time difference is $19.66 \mathrm{~ms}$. If the greatest amplitude reaches $60 \mathrm{~km}$, according to the greatest velocity of the satellite motion relative the station, the non-reciprocal error is $<290 \mathrm{ps}$.

\section{S1 S2}

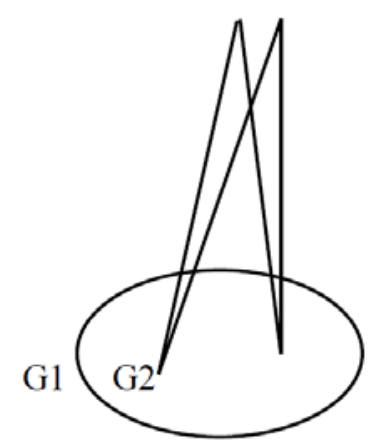

FIGURE I. THE SATELLITE RECEIVING SIGNAL AT DIFFERENT POSITIONS



G2

\section{FIGURE II. GREATEST DISTANCE DIFFERENCE FROM TWO STATIONS TO SATELLITE}

Table II shows such results of the non-reciprocities in some links that include TL-NICT, KRISS-NICT, SPRING-NICT, and NTSC-NICT, It is easy to conclude when choosing a satellite for which both elevations are about the same, which can minimize the effect; or the effect can be eliminated by offsetting the transmission time.

\section{TABLE II. NON-RECIPROCITIES DUE TO DISTANCE DIFFERENTS}

\begin{tabular}{|l|c|c|}
\hline \multicolumn{1}{|c|}{ Links } & $\begin{array}{c}\text { Distance } \\
\text { differences[km] }\end{array}$ & $\begin{array}{c}\text { Non-reciprocities[ps] } \\
\text { (amplitude: } 60 \mathrm{~km} \text { ) }\end{array}$ \\
\hline TL-NICT & 31 & $<1.6$ \\
\hline KRISS-NICT & 441 & $<27.4$ \\
\hline SPRING-NICT & 745 & $<36$ \\
\hline NTSC-NICT & 1238 & $<60$ \\
\hline
\end{tabular}

Since the two distances from two stations to the satellite are different, the time taken by the two signals from two stations to reach the satellite is different. For example, for NTSC-NMIJ link the signal from NMIJ arrives at the satellite about $4.1 \mathrm{~ms}$ before the signal from the NTSC, and for the SPRING-NICT link the NICT signal arrives about $2.5 \mathrm{~ms}$ earlier. Due to the satellite motion relative to the earth surface the satellite receives the two signals at different positions, therefore, the path delay can not be completely canceled in TWSTFT, and the non-reciprocity delay produced equals the time difference by multiplying the relative velocity of the satellite. Figure 1 and Figure 2 show the non-reciprocity delays in the two Asian links, and the daily-periodicity variation of about 20 ps is introduced into NTSC-NMIJ link and 12 ps is introduced into SG-NICT link。

Due to the Earth's rotation and the finite signal velocity, the non-reciprocal paths are produced. The effect on TWSTFT is called Sagnac effect, which can be calculated by following formula [3].

$$
E=\frac{2 \omega A}{C^{2}}
$$

where $\omega$ is the rate of Earth's rotation, $C$ is the velocity of light, $A$ is the equatorial projection of the area of the quadrangle the vertices of which are the center of the Earth and the positions of the stations on the surface of the Earth, and the position of the satellite with respect to the Earth's surface. It is thus a function of the positions of two stations and the satellite. The extreme case is about 420 ns for two 
stations at the equator, with each having an elevation of $6^{\circ}$, causing a maximal separation in longitude.

For the link of NTSC-NICT, the value of the Sagnac effect is $85.7 \mathrm{~ns}$, and the effect coefficients of longitude, latitude and altitude of the satellite variation are approximately $66 \mathrm{ps} / 0.1^{\circ}$, $0.13 \mathrm{ps} / 0.1^{\circ}$ and $2 \mathrm{ps} / \mathrm{km}$, respectively. Table III shows such results of the non-reciprocities due to the Sagnac effect in some links. It shows that the errors primarily depend on the variation of the satellite longitude and altitude. Based on the extent of the satellite motion from the satellite control center (the longitude is within $150^{\circ} \mathrm{E} \pm 0.05^{\circ}$, the latitude is within 0 ${ }^{\circ} \pm 0.05^{\circ}$, and the aviation of altitude is $<60 \mathrm{~km}$ ), the error from the Sagnac effect is at order of 100 ps in several links.

\begin{tabular}{|c|c|c|c|c|}
\hline Links & $\begin{array}{c}\text { Sagnac } \\
\text { effect } \\
\text { [ns] }\end{array}$ & $\begin{array}{c}\text { Variation } \\
\text { from } \\
\text { longitude } \\
{\left[\begin{array}{c}\sim \\
\text { ps } / 0.1 \\
\left.{ }^{\circ}\right]\end{array}\right.}\end{array}$ & $\begin{array}{c}\text { Variation } \\
\text { from } \\
\text { latitude } \\
{\left[\sim \mathrm{ps} / 0.1^{\circ}\right]}\end{array}$ & $\begin{array}{l}\text { Variation from altitude } \\
\qquad[\sim \mathrm{ps} / \mathrm{km}]\end{array}$ \\
\hline $\begin{array}{l}\text { TL-NIC } \\
\mathrm{T} \\
\end{array}$ & 63.83 & 2 & 0.10 & 1.6 \\
\hline $\begin{array}{l}\text { KRISS- } \\
\text { NICT }\end{array}$ & 34.54 & 23 & 0.05 & 0.8 \\
\hline $\begin{array}{l}\text { SPRING } \\
\text {-NICT }\end{array}$ & 125.13 & 40 & 0.20 & 3.0 \\
\hline $\begin{array}{l}\text { NTSC- } \\
\text { NICT }\end{array}$ & 85.70 & 66 & 0.13 & 2.0 \\
\hline
\end{tabular}

III. CONCLUSIONS

TWSTFT, employing a commercial communication satellite, is the only used technique allowing a remote time-transfer with accuracy potential being below 1ns. A main factor affecting the accuracy is the satellite motion. Errors, of two aspects, result from the satellite motion. One is that since the two distances from two stations to the satellite are different, the satellite receives the two signals at different position, and the non-reciprocities are produced. The largest non-reciprocal error is less than $50 \mathrm{ps}$ in the links of Asia-Pacific region. Another is that due to the Earth's rotation and the finite signal velocity, the non-reciprocal paths are also produced. We established that the variation primarily results from the satellite longitude and altitude, and the error from the Sagnac effect, is at order of 100 ps in these links.

\section{ACKNOWLEDGMENT}

This work is supported by Science and Technology Plan Projects of Xian ( No. CXY1352WL27) and Natural Science Basic Research Plan in Shaanxi Province (No. 2014JM2-1009), and the author thanks the organizations.

\section{REFERENCES}

[1] J. A. Davis, 'European two-way satellite time transfer experiments using the INTELSAT satellite at 307E', IEEE Transactions on Instrumentation and

[2] Measurement, Vol.44, No.2, 1995, pp.90-93

[3] Michto Imae, 'Two-way satellite time and frequency transfer network in pacific rim region', IEEE Transactions on Instrumentation and Measurement, Vol.50, No.2, 2001, pp.559-562

[4] E. J. Post, 'Sagnac effect', Reviews of Modern Physics, vol.39, No.2, 1967, pp.475-493. 